UCSD/PTH 92-22

June 1992

\title{
The Finite Temperature Phase Diagram of a U(1) Higgs-Yukawa Model
}

\author{
Karl Jansen \\ University of California at San Diego \\ Department of Physics-0319 \\ La Jolla, CA 92093-0319 \\ USA
}

\begin{abstract}
The finite temperature phase diagram of a U(1) Higgs-Yukawa model at a finite value of the scalar self coupling $\lambda$ is investigated by means of a large- $N_{f}$ calculation and numerical simulations. The phase diagram is similar to the one at zero temperature and shows a ferromagnetic, two symmetric and an antiferromagnetic phase. However, the phase transition lines are shifted to larger values of the Yukawa coupling demonstrating the occurence of the finite temperature symmetry restoration.
\end{abstract}




\section{Introduction}

The finite temperature electroweak phase transition plays an important role in scenarios of the early universe which assume that at a high enough temperature the universe was in a hot symmetric state [1]. During its expansion the universe cooled down and at a critical temperature passed through the electroweak phase transition breaking the symmetry spontaneously. That the symmetry should be restored at high enough temperatures was already predicted in the work of Kirzhnits and Linde [2]. A subsequent analysis [3, 4] within the framework of large $N$ and perturbative approximations confirmed the symmetry restoration picture. To test the validity of these proximations, it would be useful to investigate the phenomenon of symmetry restoration also non-perturbatively. Moreover, questions like the order of the phase transition or the value of the critical temperature $T_{c}$ can not be answered reliably in perturbation theory alone. Nonperturbative lattice simulations may provide more insight into the nature of the electroweak phase transition. However, the inclusion of all degrees of freedom is too demanding with present day computers and one has to restrict oneself to subsystems of the standard model like gauge-Higgs systems [5] or pure scalar theories. In particular in the $\mathrm{O}(4)$ symmetric $\phi^{4}$ theory the critical temperature can be estimated assuming that the gauge and fermionic degrees of freedom can be neglected. As the gauge coupling is rather weak this seems to be a reasonable approximation and one finds $T_{c} \approx 350 \mathrm{GeV}$ [6].

In this letter I want to include the fermions by studying a U(1) Higgs-Yukawa system in the large fermion number $\left(N_{f}\right)$ approximation in combination with lattice simulations. HiggsYukawa models on the lattice have been the object of numerous analytical and numerical studies in the last years [7]. They are expected to provide insight into non-perturbative properties of the Standard model. They revealed, for example, a surprisingly complex phase diagram. For a finite value of the scalar self coupling $\lambda$ four different phases were found at zero temperature [8, 9]. These phases are a ferromagnetic phase (FM), where the symmetry is spontaneously broken, two symmetric phases (SYM), one at weak and the other at strong Yukawa coupling and an antiferromagnetic (AFM) phase where the staggered magnetization is non-zero with two regions, separated by a first order phase transition line. For an infinite scalar self coupling $\lambda=\infty$ even an additional phase was found, a ferrimagnetic (FI) phase where the magnetization as well as the staggered magnetization is non-zero [10].

At small scalar self coupling $\lambda$ the system can be analyzed by analytical means like large$N_{f}$, perturbative or mean-field approximations. The non-perturbative simulation results are in good agreement with these analytical calculations [9]. In particular it could be confirmed that the phase transition from the ferromagnetic to the symmetric phase at weak Yukawa coupling, which is relevant for the standard model, is second order with the critical behaviour of the Gaussian fixed point. This means the triviality of the Higgs Yukawa models. Of course, the model is plaqued by the appearance of doubler fermions so that one can not expect quantitative results from these studies. Nevertheless, Higgs-Yukawa models are expected to describe qualitative features correctly like the order of the phase transitions and the question of symmetry restoration which I want to study here. 


\section{The model and the techniques}

\subsection{Zero temperature}

The lattice action for the $U(1)$ chiral invariant Higgs-Yukawa model at zero temperature is defined as

$$
S=S_{f}+S_{H}
$$

The fermion part of the action $S_{f}$ is given by

$$
S_{f}=\sum_{x, z} \bar{\psi}_{i}(x) M(x, z) \psi_{i}(z) \quad, \quad i=1,2, \ldots, N_{f} / 2
$$

In eqn. (2) the fermion matrix may be written as

$$
M(x, z)=\sum_{\mu} \gamma_{\mu}\left[\delta_{x+\mu, z}-\delta_{x-\mu, z}\right]+y\left[\phi_{1}(x)+i \gamma_{5} \phi_{2}(x)\right] \delta_{x, z}
$$

where $\gamma_{\mu}, \gamma_{5}$ are the Hermitian Dirac matrices and $y$ stands for the Yukawa coupling. The scalar part of the action $S_{H}$ in eqn. (1) is given by

$$
\begin{aligned}
S_{H} & =-\kappa \sum_{x, \mu} \phi_{a}(x)\left[\phi_{a}(x+\mu)+\phi_{a}(x-\mu)\right]+\sum_{x} \phi_{a}(x)^{2} \\
& +\sum_{x} \lambda\left[\phi_{a}^{2}(x)-1\right]^{2}, \quad a=1,2 .
\end{aligned}
$$

The scalar fields $\phi$ are complex, $\lambda$ is the scalar self coupling and $\kappa$ is the hopping parameter which is related to the mass parameter $m_{c}$ in the continuum formulation, $m_{c}^{2}=(1-2 \lambda-$ $8 \kappa) / \kappa$. The sums in eqs.(2) and (4) run over the lattice size $\Lambda=L_{T} L_{s}^{3}$.

The model defined in eqns.(1-田) can be solved in the large fermion number $\left(N_{f}\right)$ limit. It is convenient [9] to consider a modified form of the scalar action

$$
\begin{aligned}
S_{H} & =-\kappa_{N} \sum_{x, \mu} \varphi_{a}(x)\left[\varphi_{a}(x+\mu)+\varphi_{a}(x-\mu)\right]+\sum_{x} \varphi_{a}(x)^{2} \\
& +\lambda_{N}\left(\varphi_{a}^{2}(x)-N_{f}\right)^{2} .
\end{aligned}
$$

The usual lattice action eqns.(1-4) is obtained by identifying

$$
\kappa_{N}=C^{2} \kappa, \lambda_{N}=C^{4} \lambda, y_{N}=C y,
$$

where the factor $C$ satisfies the equation

$$
C^{4}-\left(1-2 \lambda_{N} N_{f}\right) C^{2}-2 \lambda_{N}=0
$$

The scalar field $\varphi_{a}(x)$ is related to the original field $\phi_{a}(x)$ by

$$
\varphi_{a}(x)=\phi_{a}(x) / C
$$


In the $1 / N_{f}$ expansion the couplings $\tilde{y}_{N}=\sqrt{N_{f}} y_{N}, \tilde{\lambda}_{N}=N_{f} \lambda_{N}$ are kept fixed to be $O(1)$. As $N_{f} \rightarrow \infty$ the relations in eqn. (6) simplify, giving

$$
\kappa=\frac{\kappa_{N}}{1-2 \tilde{\lambda}_{N}}, \lambda N_{f}=\frac{\tilde{\lambda}_{N}}{\left(1-2 \tilde{\lambda}_{N}\right)^{2}}, y \sqrt{N_{f}}=\frac{\tilde{y}_{N}}{\sqrt{1-2 \tilde{\lambda}_{N}}}, \quad \tilde{\lambda}_{N}<\frac{1}{2}
$$

In the large $N_{f}$ limit the constant mode of the scalar field dominates the path integral which suggests the Ansatz

$$
\varphi_{1}(x)=\sqrt{N_{f}}\left[\left(a+(-1)^{\sum_{\mu} x_{\mu}} b\right], \varphi_{2}(x)=0,\right.
$$

where $\sqrt{N_{f}} a$ and $\sqrt{N_{f}} b$ correspond to the magnetization and staggered magnetization, respectively. The effective potential at leading order is

$$
\frac{1}{N_{f}} V_{e f f}(a, b)=-8 \kappa_{N}\left(a^{2}-b^{2}\right)+\left(a^{2}+b^{2}\right)+\tilde{\lambda}_{N}\left(\left(a^{2}+b^{2}-1\right)^{2}+4 a^{2} b^{2}\right)-2 I(a, b, 0)
$$

where the zero temperature lattice integral is

$$
I(a, b, 0)=\int \frac{d^{4} k}{(2 \pi)^{4}} \log \left[\sum_{\mu} \sin ^{2} k_{\mu}+\tilde{y}_{N}^{2}\left(a^{2}-b^{2}\right)\right] .
$$

\section{$2.2 \quad$ Finite temperature}

A finite temperature on the lattice can be realized by a finite extension $L_{T}$ in the temporal direction. The other directions on the lattice are kept large enough so that finite size effects are negligible. This leads to the usual finite temperature geometry of the lattice $L_{s}^{3} L_{T}$ with $L_{s} \gg L_{T}$. The physical temperature is given in terms of the lattice spacing $a$

$$
T=1 / L_{T} a
$$

Varying the physical temperature can be achieved by changing $L_{T}$. Symmetry restoration on the lattice can then be detected in the following way: Assume that there is a phase transition from a symmetric to a broken phase at zero temperature. If now by heating the system, i.e. decreasing $L_{T}$, this phase transition shifts into the broken phase, we have found the symmetry restoration. Fixing the parameters of the theory, characterized by, for example, the renormalized scalar self coupling $\lambda_{r}$ and the renormalized Yukawa coupling $y_{r}$, to be in the broken phase at zero temperature, the system passes the finite temperature phase transition at some $L_{T}$ corresponding to a critical physical temperature $T_{c}$ and for values of $T>T_{c}$ the system is in the symmetric phase.

The lattice action of the finite temperature U(1) Higgs-Yukawa model is the same as in eqs.(1-田). The only change is that now the sums run over the finite temperature lattice with $L_{s} \gg L_{T}$. The steps of the large- $N_{f}$ approximation are therefore very similar to the zero 
temperature case. The only change is that at finite temperature the expression of $I(a, b, 0)$, eq. (12), has to be replaced by

$$
I\left(a, b, L_{T}\right)=\frac{1}{L_{T}} \sum_{n} \int \frac{d^{3} k}{(2 \pi)^{3}} \log \left[k_{4}^{2}+\sum_{i} \sin ^{2} k_{i}+\tilde{y}_{N}^{2}\left(a^{2}-b^{2}\right)\right] ; i=1,2,3
$$

where $k_{4}=2 \pi(n+1) / L_{T}, n=0, \ldots, L_{T}-1$. Note that in contrast to the continuum the sum over $n$ is finite and can not be done explicitely.

Replacing $I(a, b, 0)$ by $I(a, b, T)$ in (11) gives the finite temperature effective potential. One may obtain different phases correponding to the locations of the minima of $V_{\text {eff }}$.

(1)Symmetric (SYM) solution: There is a single minmum at $a=b=0$.

(2)Ferromagnetic (FM) solution: There is a minimum at $a \neq 0, b=0$

(3)Antiferromagnetic (AFM) solution: The minimum is at $a=0, b \neq 0$

(4)Ferrimagnetic (FI) solution: The minimum is at $a \neq 0, b \neq 0$. It can be shown that this solution does not exist for small values of the scalar self coupling $\lambda \lesssim 1$.

To determine the phase structure I have calculated the integral (14) which is needed for the effctive potential (11) numerically. I used mainly finite lattice sums as approximations to save computertime. However, I checked for various points that the lattice integrals give compatible results.

As described in [11, 8, 9], for large $y$ values another type of large $N_{f}$ expansion is possible. Here one keeps the Yukawa coupling $y_{N} \sim O\left(\sqrt{N_{f}}\right)$ and $\kappa_{N} \sim O\left(1 / \sqrt{N_{f}}\right)$. As a result of this large- $N_{f}$ expansion one obtains an effective action which is the $X Y$-model in four dimensions

$$
S_{e f f}=-\kappa_{e f f} \sum_{x, \mu} \sigma_{a}(x)\left[\sigma_{a}(x+\mu)+\sigma_{a}(x-\mu)\right]
$$

where the fields $\sigma_{a}(x)$ have unit length, $\sigma_{a}(x) \sigma_{a}(x)=1$. The effective hopping parameter $\kappa_{e f f}$ is given by

$$
\kappa_{e f f}=\kappa_{N} \varphi_{0}^{2}+\frac{N_{f}}{2 y_{N}^{2} \varphi_{0}^{2}}
$$

At finite temperature the steps of the calculation are the same with $\kappa_{e f f}$ replaced by the one of the XY-model at finite temperature, $\kappa_{e f f}(T)$. I determined $\kappa_{c}(T)$ for the XY-model on lattices with $N_{T}=6$ and $N_{T}=8$ in time direction by a numerical simulation using the cluster algorithm. I find the phase transition still to be of second order and $\kappa_{c}(T)$ shifted only slighly to larger $\kappa$-values. This shift is less than $1 \%$ similar to case of the $\mathrm{O}(4)$ model [6]. Therefore the phase transition lines from the large $N_{f}$ expansion in the strong Yukawa coupling region show only a tiny shift. 


\section{$2.3 \quad$ Numerical simulation}

I have performed numerical simulations at $\lambda=0.0156, N_{f}=2$. Note that these parameters are the same as the ones used in Fig.2a of ref. [9] for zero temperature. The Hybrid Monte Carlo method [12] was used for the dynamical fermion simulations. Each molecular dynamics trajectory consists of 10 steps with step size chosen such that the acceptance rate is around $80 \%$. As a check I did simulations at $\lambda=0.0156$ for $N_{f}=10$ and found agreement with the large- $N_{f}$ expansion. To decide the order of the phase transition, I looked for hysteresis effects in the thermocycles. For each data point in the thermocycle about 50 trajectories are used as warmup and 100-200 trajectories are used in the measurement.

The order parameters to detect the phasetransitions have been the magnetization $v$ defined as

$$
v=<\sqrt{\bar{\phi}_{a}^{2}}>, \quad \bar{\phi}_{a}=\frac{1}{L^{4}} \sum_{x} \phi_{a}(x)
$$

and the staggered magnetization $v_{s t}$ given by

$$
v_{s t}=<\sqrt{\bar{\phi}_{s t, a}^{2}}>, \quad \bar{\phi}_{s t, a}=\frac{1}{L^{4}} \sum_{x}(-1)^{\sum_{\mu} x_{\mu}} \phi_{a}(x),
$$

where $L$ is the linear size of the lattice. The measurements are done on $8^{3} 2$ and a few on $10^{3} 2$ lattices. Although these lattices are certainly not sufficient to distinguish second order and weakly first order transitions, the combination and the agreement of the numerical and analytical results give a reliable determinination of the order of the phase transitions.

\section{Discussion and conclusion}

The results of the large $N_{f}$ and Monte Carlo calculations are shown in fig.1 for the Yukawa coupling $y \leq 1.5$. I have left out the results for the strong Yukawa region as they are indistinguishable from the zero temperature case. I checked explicitely also in this region that the Monte Carlo data agree with the large $N_{f}$ predictions.

In the figure the large- $N_{f}$ results are shown as lines, where full lines indicate second order and dashed lines first order phase transitions. The Monte Carlo results for the phase transitions are exhibited as circles where open symbols mean first order and full symbols second order phase transitions. I find agreement between the Monte Carlo results and the theoretical prediction from the large $N_{f}$ calculation not only for the position but also for the order of the phase transitions. There occur three different phases, a ferromagnetic (FM), a symmetric (SYM) and an antiferromagnetic (AFM) phase. As mentioned earlier, at large values of the Yukawa coupling a second symmetric phase appears, which is not shown in fig.1. The dotted lines in fig.1 indicate the phase diagram at zero temperature as found in [9]. Although the structure and the order of the phase transitions remain the same at finite temperature, a clear shift to larger values of the Yukawa coupling is seen. 
Let me concentrate on the phase transition between the ferromagnetic (FM) and the symmetric (SYM) phases which is relevant for the standard model. As discussed above, the observed shift to larger values of the Yukawa coupling indicates the expected symmetry restoration: A point (denoted as the star in fig.1) chosen to be in the broken (FM) phase at zero temperature will be found in the symmetry restored phase at finite temperature. It is noteworthy that for the moderate temperatures that could be tested here the phasetransition is still second order and in the domain of attraction of the Gaussian fixed point. I conclude that symmetry restoration is not only an effect of a large $N$ or pertubative apparoximation but survives also when non-perturbative methods are applied.

\section{Acknowledgement}

I want to thank Y.Shen for several useful discussions. This work is supported by DOE grant at UC San Diego (DE-FG-03-90ER40546).

\section{References}

[1] R.H. Brandenberger, Rev.Mod.Phys. 57 (1985) 1.

[2] D.A. Kirzhnits and A.D. Linde, Phys.Lett. 42B (1972) 471.

[3] L. Dolan and R. jackiw, Phys.Rev.D9 (1974) 3320.

[4] S. Weinberg, Phys.Rev.D9 (1974) 3357.

[5] H.G. Evertz, J. Jersák and K. Kanaya, Nucl.Phys. B285 [FS19] (1987) 229.

[6] K. Jansen and P. Seuferling, Nucl.Phys.B343 (1990) 507.

[7] J. Shigemitsu, Nucl. Phys.B (Proc. Suppl.) 20 (1991) 515; M.F.L. Golterman, Nucl. Phys.B (Proc. Suppl.) 20 (1991) 528; I. Montvay, Proceedings of the Conference on Lattice filed Theory, "Lattice '91", Tokyo (1991).

[8] A. Hasenfratz, P. Hasenfratz, K. Jansen, J. Kuti and Y. Shen, Nucl. Phys. B365 (1991) 79.

[9] A. Hasenfratz, K. Jansen and Y. Shen, UCSD preprint, UCSD/PTH 92-08.

[10] A. Hasenfratz, W.Liu and T. Neuhaus, Phys.LettB236 (1990) 339; W. Bock, A. K. De, K. Jansen, J. Jersák, T. Neuhaus and J. Smit, Nucl. Phys. B344 (1990) 207.

[11] I.K. Affleck, Phys.Lett.B109 (1982) 307.

[12] S. Duane, A. D. Kennedy, B. J. Pendleton and D. Roweth, Phys. Lett. 195B, (1987) 216. 
Fig. 1 The finite temperature phase diagram at $\lambda=0.0156$ and $N_{f}=2$. The $\mathrm{MC}$ data are indicated by circles where the solid symbols denote second and the open symbols first order phase transitions. The solid and dashed lines are the results from the $1 / N_{f}$ expansions, where the solid lines represent second order and the dashed line first order phase transitions. The phases are: (FM) ferromagnetic, (SYM) symmetric and (AFM) antiferromagnetic. The dotted lines indicate the zero temperature phase diagram [9]. Note that the phase transitions for finite temperature are shifted to larger values of the Yukawa coupling. This shows the expected finite temperature symmetry restoration as indicated by the starred point which is in the broken phase at zero and in the symmetric phase at finite temperature. 